\section{Perfect energy stability in neurons}

L. Felipe Barros and Felipe Baeza-Lehnert

Neurons invest considerable energy in the generation and maintenance of a steep electrochemical $\mathrm{Na}^{+}$ gradient across the plasma membrane. The $\mathrm{Na}^{+}$gradient is in turn used for various housekeeping tasks, including import of nutrients, export of waste, volume and $\mathrm{pH}$ regulation, and modulation of $\mathrm{Ca}^{2+}$ signaling. In addition, neurons make intensive use of the $\mathrm{Na}^{+}$ gradient to transmit information by membrane depolarization, to the extent that in these cells the $\mathrm{Na}^{+} / \mathrm{K}^{+}$ATPase pump accounts for over $80 \%$ of the ATP turnover. Almost all ATP is produced in mitochondria, so how does the organelle know how much to produce and when? This is a central question in bioenergetics. Ever since the studies of isolated mitochondria by Britton Chance and colleagues in the hay days of cybernetics and feedback control theory, the coupling between energy demand and supply has been assigned to ADP [1]. Later on Krebs cycle dehydrogenases and other mitochondrial enzymes were found to be sensitive to $\mathrm{Ca}^{2+}[2,3]$, suggesting an additional coupling mechanism.

Now is the era of genetically-encoded fluorescent sensors, which afford improved spatial and temporal resolution for the study of metabolism in intact cells [4]. Equipped with these tools, our group set out to map the first seconds of the neuronal response to workload [5]. The initial results were ostensibly boring. Neuronal ATP, ATP/ADP, glucose, pyruvate, lactate and $\mathrm{pH}$ were all found to be unaffected by excitatory neurotransmission. However, flux measurements using transporter-stop protocols unveiled a rich underlying phenomenology. Neuronal ATP consumption had been stimulated by $200 \%$, while glycolytic flux and mitochondrial pyruvate consumption had increased by $200-300 \%$. The stability was therefore explained not by lack of demand but by a close second-to-second match between ATP consumption and production. In vivo determinations in the somatosensory cortex confirmed the stability of neuronal ATP through bouts of activity. How could ADP or ATP possibly play any regulatory role if their concentration does not change? Next we turned to the alternative hypothesis but found the cytosolic $\mathrm{Ca}^{2+}$ had already returned to resting level at the time of maximum ATP turnover, and that intramitochondrial $\mathrm{Ca}^{2+}$ did not even rise. Further experiments indicated that the $\mathrm{Na}^{+}$pump itself controls mitochondria, acting via an unspecified mechanism that may involve the glycolytic machinery [5].

The inordinate metabolic stability of neurons is exciting. Is neuronal ATP depletion a reflection of high level physiological stimulation or is it frankly pathological? In support of the latter, both tetanic stimulation and glutamate impaired mitochondrial pyruvate consumption, showing that the ATP depletion is partly secondary to a drop in production [5]. ATP depletion affects scores of reactions and under certain conditions may trigger a runaway metabolic collapse [6]. Neurons must endure a lifetime of unpredictable activations without the benefit of energy stores. Could repetitive cycles of ATP depletion over years of dysfunctional adaptation contribute to neurodegeneration? The machinery that links the $\mathrm{Na}^{+}$pump to mitochondria to ensure perfect energetic stability in neurons may hold clues to these important questions.

\section{REFERENCES}

1. Chance B, Williams GR. J Biol Chem. 1955; 217:409-28. http://www.jbc.org/content/217/1/409.short PMID:13271404

2. McCormack JG, et al. Physiol Rev. 1990; 70:391-425. https://doi.org/10.1152/physrev.1990.70.2.391 PMID:2157230

3. Satrústegui J, et al. Physiol Rev. 2007; 87:29-67. https://doi.org/10.1152/physrev.00005.2006 PMID:17237342

4. Barros LF, et al. Glia. 2018; 66:1138-59. https://doi.org/10.1002/glia.23248 PMID:29110344

5. Baeza-Lehnert F, et al. Cell Metab. 2019; 29:668680.e4. https://doi.org/10.1016/j.cmet.2018.11.005 PMID:30527744

6. Le Masson, et al. Neuron. 2014; 83:975-88. https://doi.org/10.1016/j.neuron.2014.07.001 PMID:25088365

L. Felipe Barros: Centro de Estudios Científicos - CECs, Valdivia, Chile

Correspondence: L. Felipe Barros

Email: fbarros@cecs.cl

Keywords: homeostasis, ATP/ADP, Na+/K+ ATPase pum, glycolysis, oxidative phosphorylation 
Acknowledgements: We thank Karen Everett for critical reading of the manuscript

Funding: The Centro de Estudios Científicos (CECS) is funded by the Chilean Government through the Centers of Excellence Basal Financing Program of CONICYT

Copyright: Barros and Baeza-Lehnert. This is an open-access article distributed under the terms of the Creative Commons Attribution License (CC BY 3.0), which permits unrestricted use, distribution, and reproduction in any medium, provided the original author and source are credited

Received: August 10, 2019

Published: September 9, 2019 